\title{
Comparison of a vertical electric and a vertical magnetic source for cross well CSEM monitoring of $\mathrm{CO}_{2}$ injection \\ Brett Harris* and Andrew Pethick, Curtin University, Australia
}

\section{Summary}

Controlled source electromagnetic transmitters create highly geometric coupled electric and magnetic vector fields that propagate in a way that is dependent on both the orientation of the transmitter and electrical conductivity distribution. There may be a good case for using cross well controlled source electromagnetic methods for monitoring injection of $\mathrm{CO}_{2}$ into deep saline or brackish sandstone reservoirs. The expected range of geo-electrical frameworks that can be used to represent $\mathrm{CO}_{2}$ injection into a saline or brackish sandstone water saturated reservoir is reasonable constrained. That is injection of $\mathrm{CO}_{2}$ would likely create an expanding zone of elevated electrical resistivity that would move out from the injector well into the reservoir. The reservoir would typically be confined above and possibly below by conductive clay or shale dominated sediments. Given this type geo-electrical framework we consider the relative merits of a time harmonic vertical electric and vertical magnetic source for monitoring $\mathrm{CO}_{2}$ injection. We compare numerically generated electric and magnetic fields created in a heterogeneous horizontally layered injection zone with and without injection of $\mathrm{CO}_{2}$. Examples are first provided for a layered earth and then for an expanding 3D volume within permeable layers. We provide images indicating that the vertical electric dipole source is sensitive to $\mathrm{CO}_{2}$ injection into thin resistive sandstone layers in a conductive background. We explore why the more common vertical magnetic dipole source is comparatively insensitive to an increase in resistivity in thin sandstone layers. In summary the vertical magnetic dipole source is a common and practical in-hole source, however in principle the vertical electrical dipole source is likely to be more suitable for monitoring $\mathrm{CO}_{2}$ injection. Certainly the use of a vertical electric dipole source would need to be facilitated within the monitoring well design. Ideally the monitoring interval should be open hole or at least the casing should be slotted, non-metallic and have considerable open area to the formation.

\section{Introduction}

Electromagnetic techniques like MT (i.e. the magnetotelluric method) and LOWTEM (i.e. the Long Offset Transient Electromagnetic method) have been identified as possible technologies for monitoring large scale carbon dioxide $\left(\mathrm{CO}_{2}\right)$ injection during sequestration. However for monitoring $\mathrm{CO}_{2}$ injection into deep saline reservoirs both methods suffer from a significant problem. That problem is transmitter and receiver distance from the injection zone. This problem was highlighted by Suryopranoto, 2009 who shows many examples of the rapidly diminishing "detectability" of electrically resistive gas filled layers with increasing depth for both LOWTEM and MT. Suryopranoto's basic conclusion was that given typical surface noise levels and the ever present reality of electrical equivalence, depths greater than $1000 \mathrm{~m}$ would be highly challenging for any surface based electromagnetic (EM) method.

The potential of surface EM measurements for monitoring $\mathrm{CO}_{2}$ injection could be significantly improved if inversion were constrained with resistivity distributions derived from cross-well, surface to hole or wire-line induction logging integrated with a geological framework developed from seismic reflection. That is, aside from independent monitoring of $\mathrm{CO}_{2}$ injection, in-hole EM methods could act as an important constraint on inversion of surface EM measurements. This is because in-hole methods should robustly express the relationship between $\mathrm{CO}_{2}$ injected and local conductivity changes in the injection zone. Considerable work remains before surface methods can claim a space in $\mathrm{CO}_{2}$ monitoring, however if any success is to be achieved it is likely that time lapse in-hole measurements with EM methods will need to play a role.

Unlike time lapse surface EM techniques, time lapse induction logging has proved valuable for monitoring both $\mathrm{CO}_{2}$ gas injection (Xue, 2006 et. al.) and in monitoring injection of low solute concentration water for aquifer recharge projects (Malajczuk, 2010). Further multifrequency tri-axial induction logging tools are now available (Wang. et. al. 2006) and present the possibility of recovering full tensor conductivity by transmitting and receiving electromagnetic fields via small coils with multiple orientations (i.e. a $3 \mathrm{C}$ magnetic dipole transmitter and receiver system).

Background or pre-injection electrical conductivity distribution for $\mathrm{CO}_{2}$ injection into a saline or brackish reservoir will likely be dominated by solute concentration distribution combined with clay/shale type and distribution. Solute concentration typically increases with depth, however the opposite can be true if basin hydrodynamic is such that lower solute concentration water is driven below clay/shale dominated sediments. However in general deep reservoirs are often highly saline and of little value as water supply. Changes in formation electrical resistivity that may be associated with injecting $\mathrm{CO}_{2}$ into a saline reservoir are examined by Zue et. al. 2006 and Kim et. al., 2010. Laboratory tests of $\mathrm{CO}_{2}$ injection into brine filled 


\section{Comparison of a vertical electric and a vertical magnetic dipole source for monitoring $\mathrm{CO}_{2}$ injection}

permeable sands tend to show greater change in resistivity than do results from time lapse induction logging. This difference is likely a result of clay content and the common use of vertical magnetic dipole (VMD) source and receiver EM logging systems (i.e. the typical induction logging tool). Such systems tend to be more "sensitive" to conductive layers. One clear weaknesses of using the VMD source for detecting resistivity layers is that it can only senses horizontal resistance to current flow if logging is completed a vertical hole penetrating horizontal layering.

Field examples of the application of a vertical electric dipole (VED) source and receiver systems are far from common and often not ideally suitable for detailed cross well monitoring of $\mathrm{CO}_{2}$ injection. Several studies consider the possibility of deploying a VED source in steel casing (e.g. Wang. et. al 2009). Many other model studies do exist (e.g. Kong et. al. 2009 and Spies and Habashy 1995).

\section{Method}

One reasonable question to ask is, how are relative "sensitivities" for the various source configurations assessed. We choose to make a direct comparison of the EM fields computed for a VED and VMD source for geoelectrical models representing resistivity distribution before and after injection of $\mathrm{CO}_{2}$. We also compare a normalized amplitude. This is the ratio of vector field amplitudes before and after injection as a percent. Injection of $\mathrm{CO}_{2}$ is geo-electrically represented by an increase in electrical resistivity in thin high permeability layers. That is, electrical resistivity increases in the layers where $\mathrm{CO}_{2}$ has displaced some fraction of the saline or brackish water.

The formulation for computing the electric and magnetic fields generated by a time harmonic dipole electromagnetic source is well known (e.g. Wait, J. R., 1970). We use open source software to compute the electromagnetic fields about different time harmonic dipole sources. These software include the general purpose 3D integral equation code Marco (AMIRA INTERNATIONAL 2011) and the layered earth code Dipole1D developed by Kerry Key (2009). These algorithms are coordinated by the java based EM data management and visualization software developed by Andrew Pethick 2011 (i.e. www.MCSEM.com).

A vertical magnetic dipole source within a horizontal layered earth will generate electrical fields circulating in the plane of the layering. That is, the electric fields generated by a VMD source have no vertical component. Conversely a VED source will generate magnetic fields that circulate in the plane of the layering. That is, for a VED source the generated magnetic fields will have no vertical component. Intuitively we should suspect that the VMD will be relatively insensitive to an increase in resistivity for resistive layers as would be expected for $\mathrm{CO}_{2}$ injection into thin high permeability sandstone layers within a more clay/shale dominated background. Our aim is to examine and illustrate the basic difference between a VMD and VED source. We do this by showing streamlines and or vector fields before and after injection for the VED and VMD source. We also provide sections showing the ratio of field amplitudes before and after injection as a percent. An example is provided below.

\section{Example}

To illustrate difference between the VED and VMD source we chose a geo-electrical model broadly based on the distribution permeable sands in the lower approximately $200 \mathrm{~m}$ of the Paaratte Formation in the OTWAY basin, Victoria Australia (i.e. see Bunch 2010). The Paaratte is a brackish reservoir and resistivity distribution can be observed from wire-line logs completed in drill hole CRC2 (Bunch, 2010). Drill hole CRC2 was completed as part of the Australian government funded CO2CRC research project. The example provided here compares electric field generated by a VED (see Figure 1) and the magnetic fields generated by the VMD (see Figure 2). The lower part of the Paaratte at CRC2 appears to be a heterogeneous mix of high permeability sandstone layers (e.g. plus $4000 \mathrm{mD}$ ) and generally lower permeability background sediments (e.g. silty, cemented and or shale dominated sediments).

We have constructed a highly simplified resistivity distribution based on nine thin high permeability layers. We group all low permeability sediments as "background" and assign a resistivity of $10 \mathrm{Ohm} . \mathrm{m}$. Higher permeability sandstone dominated layers are regarded as the "injection" intervals and are assigned a resistivity of $20 \mathrm{Ohm} . \mathrm{m}$ before injection. We assign the post injection resistivity (i.e. maximum $\mathrm{CO}_{2}$ saturation) in the thin high permeability layers of $40 \mathrm{Ohm} . \mathrm{m}$. Clearly formation complexity and the reality of $\mathrm{CO}_{2}$ injection is far more complicated, with $\mathrm{CO}_{2}$ expected to migrate and partly fill all connected porous layers. Also any change in resistivity related to $\mathrm{CO}_{2}$ injection is highly dependent on post injection connectivity of residual saline or brackish water. Again the example provided is mainly intended to highlight the difference between a VED and VMD source for monitoring $\mathrm{CO}_{2}$ injection into resistive thin sandstone layers. We compared EM fields generated at many frequencies and from many perspectives, however for this example a frequency of 1000 $\mathrm{Hz}$ is selected. We chose a relatively high frequency as monitoring should be expected to recover the detailed nature on injection. We have also considered a full range of transmitter positions however to illustrate the general principle in this example we show one position towards the middle of the injection zone (see the dipole position in Figures 1, 2 and 3). 


\section{Comparison of a vertical electric and a vertical magnetic dipole source for monitoring $\mathrm{CO}_{2}$ injection}

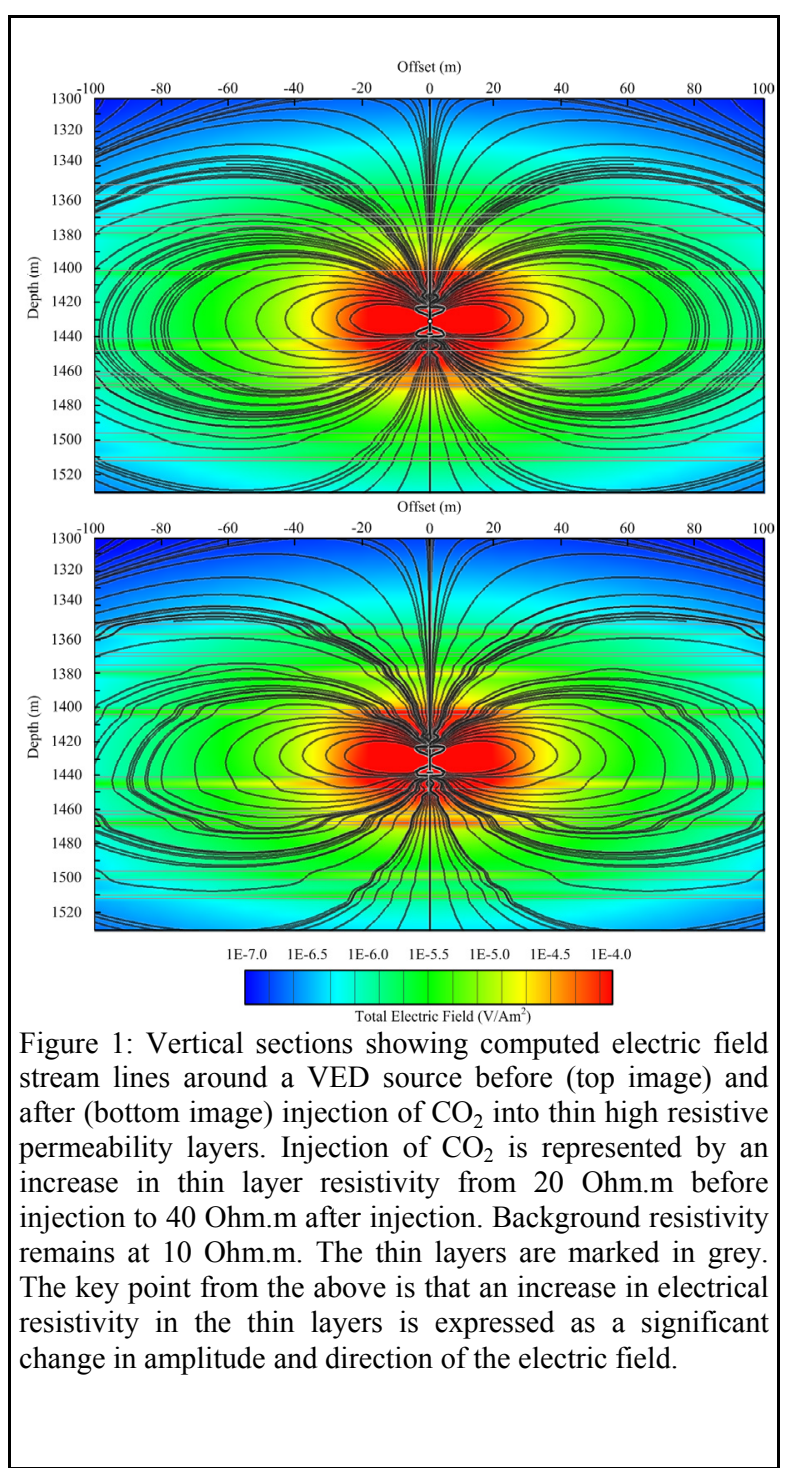

The strong impact of increasing the thin layer resistivity from 20 to $40 \mathrm{Ohm} . \mathrm{m}$ is clear from Figure 1 (see the change in amplitude and direction of the electric field in bottom image). It is also evident from the stream lines that the direction of the vertical component of the electric field is significantly changed after injection. This is important because measurement with a VED receiving antenna in a vertical open hole or through perforated non-metallic casing is a far more reasonable proposition than the measurement of the other components. Ultimately cross well inversion constrained by well logs would be used to aid in recovering changes in formation resistivity related to $\mathrm{CO}_{2}$ injection.

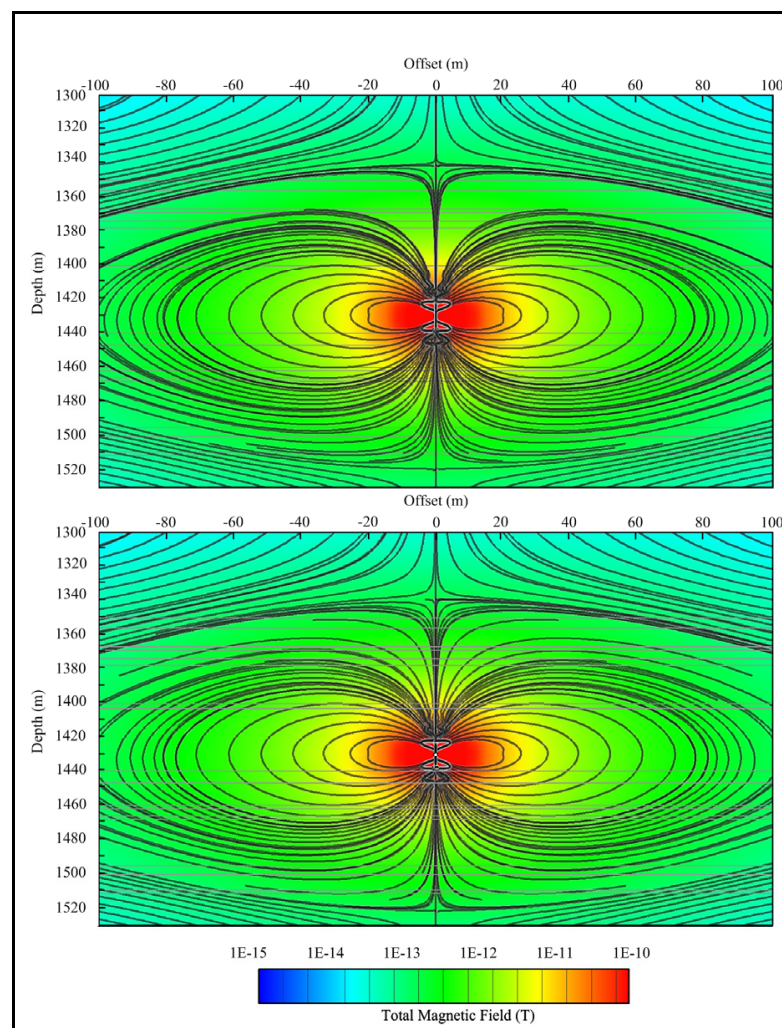

Figure 2: Vertical sections showing computed magnetic field stream lines from a VMD source before and after injection into thin high permeability layers. Injection is represented by an increase in layer resistivity from 20 Ohm.m before injection to $40 \mathrm{Ohm} . \mathrm{m}$ after injection within a $10 \mathrm{Ohm} . \mathrm{m}$ background. Thin layers are marked in grey. The key point from the above is that the thin layers have negligible impacts on amplitude and direction of the magnetic field when resistivity is increased from 20 (top image) to $40 \mathrm{Ohm} . \mathrm{m}$ (bottom image).

Figure 2 serves to highlight how poorly the magnetic fields generated by a VMD source would express an increase in resistivity of thin sandstone layers. There is only small percent difference between the images above (i.e. less than a few percent). While an increase of 20 to $40 \mathrm{Ohm} . \mathrm{m}$ for the thin layers sees the electric field amplitudes resulting from a VED increase in placed by more than $50 \%$ the magnetic fields resulting from a VMD source remain almost unaltered. We choose to make comparisons with magnetic fields generated by VMD source as this arrangement represents a commonly available cross well EM configuration. 


\section{Comparison of a vertical electric and a vertical magnetic dipole source for monitoring $\mathrm{CO}_{2}$ injection}

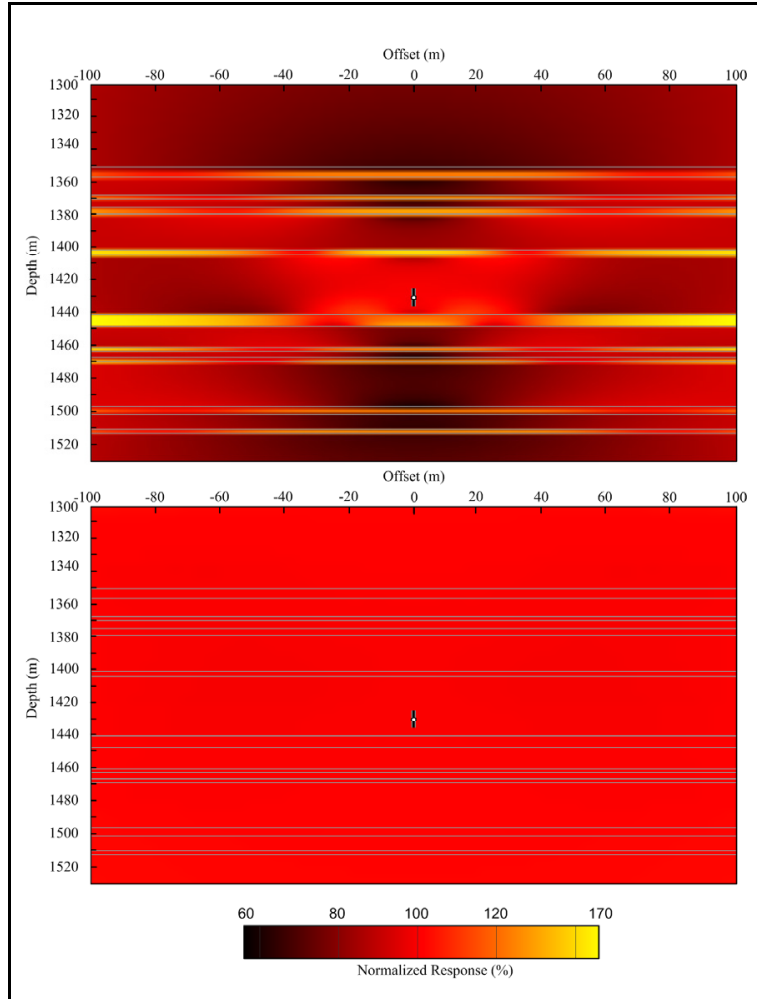

Figure 3: Top image shows the ratio of computed total electric field amplitude around a VED source before and after $\mathrm{CO}_{2}$ injection and the bottom image shows the ratio of computed total magnetic field amplitude around a VMD source before and after $\mathrm{CO}_{2}$ injection. The ratio is provided as a percent. $\mathrm{CO}_{2}$ injection is represented by an increase in layer resistivity from 20 to $40 \mathrm{Ohm} . \mathrm{m}$ in the thin layers and background resstivity is $10 \mathrm{Ohm} . \mathrm{m}$. Both images are shown with the same colours scale to highlight the significant difference in normalized responses in the thin layers.

Figure 3 is provided to highlight the significant difference between a VED source and a VMD source. The percent difference in electric field around a VEM source before and after injection is mostly large and positive in all the layers (i.e. often more that $50 \%$ ). That is time lapse changes for the VED are clear. We choose to provide an example with very thin layers for good reason. For monitoring $\mathrm{CO}_{2}$ injection the objective should be to monitor in detail how the $\mathrm{CO}_{2}$ passes from the injection well (e.g. screened or perforated interval) into the formation. Initially the injectant is expected to move out much faster in the high permeability layers than in the lower permeability layers. For the Paaratte the highest permeability layers are in places less than two meters thick. It is the rapid initial movement of $\mathrm{CO}_{2}$ into high permeability layers and subsequent filling of connected porous layers that need to be monitored. We provide clear examples of why in principle measuring electric field around a VED should be preferred over measuring the magnetic field around a VMD. Although highly problematic it is still conceivable that horizontal electric fields could be measured around a VMD source. However the problem remains that EM response is dominated by current flowing in the more conductive layers for a VMD source. That is for a vertical hole penetrating thin horizontal layering the VMD source generates current that must travel in the plane of the layering and at best electric fields can only express horizontal resistance to current flow.

\section{Conclusion}

Our work is intended to highlight the reasons why, in principle, a vertical electrical dipole source should be more suited to monitoring $\mathrm{CO}_{2}$ injection into deep thin brine saturated sandstone layers when compared with a vertical magnetic dipole source. Our direct comparison of magnetic fields generated by a VMD and the electric fields generated by a VED source in a layered medium with and without $\mathrm{CO}_{2}$ injection gives good insight into the relative merits of these two EM transmitter types. However given that only VMD cross well EM systems are in common use a full and practical comparison is problematic. That is, there are practicalities related to tool and casing design that in the short term may outweigh the theoretical benefit of one source over the other. For example the electrodes for a VED source or receiver should ideally be in direct communication with the formation. The ideal monitoring well design for a VED source and receiver system would be open hole (i.e. no casing) over the full injection interval. This is unlikely to be acceptable, so alternatively a monitoring well could be designed such that the injection interval were cased with slotted or perforated non-metallic materials.

In the long term a multi-frequency, multi-separation, multiorientation cross well EM system using electric and magnetic field transmitters should be able to provide full time lapse tensor conductivity for any conductivity distribution. For the present, steps to prototype and build new VED based EM systems for monitoring injection of $\mathrm{CO}_{2}$ into deep permeable sandstone reservoirs are proceeding.

\section{Acknowledgements}

We acknowledge the funding provided by the Australian government through the CRC Program to support CO2CRC research. 


\section{EDITED REFERENCES}

Note: This reference list is a copy-edited version of the reference list submitted by the author. Reference lists for the 2011 SEG Technical Program Expanded Abstracts have been copy edited so that references provided with the online metadata for each paper will achieve a high degree of linking to cited sources that appear on the Web.

\section{REFERENCES}

AMIRA International, 2011, P223 — EM modelling software, http://www.amirainternational.com/WEB/site.asp?section=news\&page=projectpages/p223f_softwar e, accessed 25 January 2011.

Bunch, M., 2010, Site characterization at the CO2CRC Otway Basin: Presentation at the China Australia Geological Storage Workshop II.

Key, K., 2009, 1D inversion of multi-component, multifrequency marine CSEM data: Methodology and synthetic studies for resolving thin resistive layers: Geophysics, 74, no. 2, F9-F20, doi:10.1190/1.3058434.

Kim, J., Z. Xue, and T. Matsuoka, 2010, Experimental study on C02 monitoring and saturation with combined P-wave velocity and resistivity: International Oil \& Gas Conference and Exhibition, SPE, 130284.

Kong, F. N., F. Roth, P. A. Olsen, and S. O. Stalheim, 2009, Casing effects in the sea-to-borehole electromagnetic method: Geophysics, 74, no. 5, F77-F87, doi:10.1190/1.3173807.

Malajczuk, S., 2010, Time lapse thermal and induction logging in the near well environment, Perth Basin, WA: Curtin University Report No. GPH 15/10.

Spies, B. R., and T. M. Habashy, 1995, Sensitivity analysis of crosswell electromagnetics: Geophysics, 60, 834-845, doi:10.1190/1.1443821.

Suryopranoto, B. S., 2009, Feasibility study on using electromagnetic methods for detection and monitoring of deep carbonate reservoirs, the Bunga Field, Indonesia: Curtin University Report No. GPM 13/09.

Wait, J. R., 1970, Electromagnetic waves in stratified media, 2nd edition: Pergamon Press.

Wang, H., T. Barber, K.-C. Chen, S. Davydycheva, M. Frey, and D. Homan, 2006, Tri-axial induction logging - Theory, modeling, inversion and interpretation: International Oil \& Gas Conference and Exhibition, SPE, 103897.

Xue, Z., J. Kim, S. Mito, K. Kitamura, and T. Matsuoka, 2009, Detecting and monitoring C02 with Pwave velocity and resistivity from both laboratory and field scales: International Conference on $\mathrm{CO} 2$ Capture, Storage, and Utilization, SPE, 126885.

Xue, Z., D. Tanase, and J. Watanabe, 2006, Estimation of CO2 saturation from time-lapse CO2 well logging in an onshore aquifer, Nagaoka, Japan: Exploration Geophysics, 37, no. 1, 19-29, doi:10.1071/EG06019.

Yang, W., C. Torres-Verdin, J. Hou, and Z. Zhang, 2009, 1D subsurface electromagnetic fields excited by energized steel casing: Geophysics, 74, no. 4, E159-E180. 